\title{
TDOA Ranging Based Cooperative Localization for Wireless Sensor Network
}

\author{
ZHANG Xin-rong ${ }^{1,3, a}$, CHANG Bo ${ }^{2, b}, X V$ Bao-guo $3, \mathrm{c}$ \\ ${ }^{1}$ Faculty of Automation, Huaiyin Institute of Technology, huaian, 223003, China \\ ${ }^{2}$ Faculty of Electronic Information Engineering, HuaiYin Institute of Techenology, huaian, 223003, \\ China \\ ${ }^{3}$ School of Internet of Things Engineering, Jiangnan University, wuxi, 214122, China \\ ann33@163.com, ${ }^{b} \mathrm{mmm} 33534 @$ sohu.com,
}

Keywords: TDOA measurements, cooperative localization, Cramér-Rao lower bound (CRLB), wireless sensor network.

\begin{abstract}
Measurement error is known to degrade remarkably the target localization accuracy. Cooperative localization using time difference of arrival (TDOA) measurements is a major research subject for positioning applications which is concerned by this paper to reduce the loss in localization accuracy owing to measurement error. In this paper we investigate neighborhood collaboration based cooperative localization algorithm with significantly lower computational complexity of all targets in wireless sensor network. We evidently demonstrate the superior trade-off between estimation performance and computational complexity of the proposed algorithm. The proposed iterative algorithm results incorrectly converged and the converged solution would approximately be the optimum solution when the measurement errors are sufficiently small. Simulations are used to confirm and support the theoretical development.
\end{abstract}

\section{Introduction}

Wireless sensor networks applications include target detection, environmental sensing, traffic monitoring, and many other fields [1-3]. In wireless sensor network applications, target node localization refers to the process of estimating the coordinates of target nodes based on different forms of distance measurements and using the information of a few anchors that their coordinates is known in advance. A large number of positioning system work is committed to establish CRLB as a benchmark to assess the localization accuracy [4-7].

A large number of literatures are available for node localization according to TDOAs where some methods are iterative and require good initial position setting [8], and some are closed-form location solutions [9]. Zheng and $\mathrm{Wu}$ [10] are probably the first to combine the localization and synchronization issues by a signal processing way with message exchanges, and proposed a constrained weighted least-squares (CWLS) estimator that has less calculation than the Maximum-Likelihood estimator (MLE).Reference [11] further heightened the sequential algorithms from [12] by introducing pseudo anchor position refinement scheme that improves the pseudo anchor positions for better locating the target nodes. However, the method from[11] can be unable to obtain the CRLB estimation accuracy when the pseudo anchor positions do not have independent estimation errors. Moreover, this joint estimation algorithm is based on ML estimation and Monte Carlo based method which are iterative and require reasonably accurate initialization. There are communications between nodes in collaborative localization [13], hence the time measurements from message exchanges among nodes can contribute to improve the estimation accuracy and reduce error accumulation in the WSN position update but usually with high computational complexity. In this paper we propose a new iterative localization algorithm according to ranging implements positioning solution in a computationally efficient way when the measurement errors are sufficiently small, the converged solution would approximately reach the correct positions of all target nodes.

We organize the paper as follows. In Section II described positioning scenario and problem 
formulation. The new iterative localization algorithm is proposed in Section III. Sections IV examined the performance evaluation of the proposed algorithm. Simulation results are provided in Section V to corroborate the theoretical derivations. Section VI concludes the paper.

\section{Location Modeling and Problem Formulation}

Considering a set of $N$ target nodes $\mathrm{S}=\left\{s_{1}, s_{2}, \cdots, s_{N}\right\}$, randomly deployed in a two dimensional(i.e., 2-D) positioning area. The true position of $s_{i}$ is given by a unknown vector $z_{i}=\left[z_{x i}, z_{y i}\right]^{T}$ whose estimated position is another vector $p_{i}=\left[p_{x i}, p_{y i}\right]^{T}$ to be determined. In addition, there are $M$ anchors $A=\left\{a_{1}, a_{2}, \cdots, a_{M}\right\}$ with GPS or any other location method randomly deployed in a WSN. The true position of an anchor $a_{k}$ known in advance is given by a vector $q_{k}=\left[q_{x k^{\prime}}, q_{y k}\right]^{T}$. A target node at unknown position $s_{i}$ radiates a signal that is captured by anchora $_{k}$. Assume the known signal propagation speed is, we have the distance measurements

$$
R_{k 1}^{i}=d_{k 1}^{i}+v_{k 1}^{i}
$$

Where $R_{k 1}^{i}=R_{k}^{i}-R_{1}^{i}, d_{k 1}^{i}$ is the true TDOA-based distance between target nodes $s_{i}$ and anchor pair $a_{k}$ and $a_{1}, d_{k 1}^{i}=c t_{k 1}^{i}, t_{k 1}^{i}$ is the true TDOA, $v_{k 1}^{i}$ mean random distance measurement noise for each time, and

Where $\|*\|$ is the Euclidean norm.

$$
d_{k 1}^{i}=d_{k}^{i}-d_{1}^{i}=\left\|p_{i}-q_{k}\right\|-\left\|p_{i}-q_{1}\right\|
$$

The range estimation between a target node $s_{i}$ and an anchor $a_{k}$ can be denoted as

$$
R_{i k}=R_{k i}=d_{i k}+e_{i k}
$$

Where $d_{i k}=\left\|z_{i}-q_{k}\right\|$, and $e_{i k}$ represents the random distance measurement error introduced by complex wireless communication environment and the ranging technique.

The node localization is to determine the positions of the target nodes $s_{i}$, as accurately as possible by exploiting the noisy $R_{i k}$. In other words, The objective is to obtain the target node position $p_{i}$ based on the TDOA measurements from $s_{i}$ and from $a_{k}$.

\section{The Proposed Cooperative Localization Algorithm}

We assume that all nodes implement common iterative self-positioning algorithm, namely, every node updates its estimated position based on locally collected information.

The Proposed Positioning Algorithm. In each iteration of the algorithm, the current estimated position of this node is updated on the basis of its neighbors to produce same number of new estimations. Therefore, when the current estimated position of the node is updated according to its neighbor, there are two kinds of cases.

CaseI: when the calculated distance, namely, the distance between the current estimated position of the node and the position or the estimated position of this neighbor, is no farther than the measured distance $R_{i j}$, namely, the obtained measurement on the distance between the node and the neighbor, we can have the new estimation according to its $j$-th neighbor given by

$$
\begin{aligned}
& \hat{p}_{i}^{(j)}(n+1)=\hat{p}_{i}(n) \\
& \quad+\frac{\hat{p}_{i}(n)-b_{j}(n)}{\left\|\hat{p}_{i}(n)-b_{j}(n)\right\|}\left(R_{i j}-\left\|\hat{p}_{i}(n)-b_{j}(n)\right\|\right)
\end{aligned}
$$

Where $b_{j}(n)$ is position $q_{k}$ of neighbor anchor or position estimation $\hat{p}_{j}(n)$ of the $j$-th neighbor of node $s_{i}$ at time $n$. By averaging these new estimations according to all its neighbors, the updated estimated position of the node is then given by 


$$
\hat{p}_{i}(n+1)=\frac{1}{N_{i}} \sum_{j \in s_{i}} \hat{p}_{i}^{(j)}(n+1)
$$

Where $N_{i}$ is the size of set $S_{i}$, and $S_{i}$ is the set containing all neighbors of nodes $s_{i}$.

Case II: otherwise, namely, $\left\|\hat{p}_{i}(n)-b_{j}(n)\right\|>R_{i j}$, the estimated position of the node has a new estimation, hence the newly calculated distance equals to the measured distance. We denote the current estimated position of nodes $s_{i}$ at time $n$ as $\hat{p}_{i}(n)$, so that we can have the new estimation according to its $j$-th neighbor as follow.

$$
\begin{aligned}
\hat{p}_{i}^{(j)}(n+1) & =\hat{p}_{i}(n) \\
& \quad-\frac{\hat{p}_{i}(n)-b_{j}(n)}{\left\|\hat{p}_{i}(n)-b_{j}(n)\right\|}\left(\left\|\hat{p}_{i}(n)-b_{j}(n)\right\|-R_{i j}\right)
\end{aligned}
$$

Similarly, by averaging these new estimations according to all its neighbors, the updated estimated position of the node is then given by

$$
\hat{p}_{i}(n+1)=\frac{1}{N_{i}} \sum_{j \in S_{i}} \hat{p}_{i}^{(j)}(n+1)
$$

We can see that the updating operation of each node in each iteration is relatively simple.

Initialization Technology. When the initialization is implemented on each individual node, there are four cases as follows. Ideally, when the target node has three or more neighbor anchors, the initial estimation on the position of this node can be calculated or estimated with least square criterion by one of the existing algorithms for ranging-based positioning, such as the one proposed in [5] and[16].The less likely scenario is that when the target node has two neighbor anchors, the initial estimation can be set as their average value of the two possible solutions. Unfortunately, when the target node has one neighbor anchor, the initial estimation can be set as the position of this anchor. Most unfortunately, when the target node has no neighbor anchor, the initial estimation can be set as the average of the initial or updated estimations on the positions of its neighbor nodes that has been initial estimated.

Algorithm Summary. The proposed algorithm is summarized in Algorithm I.

\begin{tabular}{l}
\hline Algorithm I the proposed algorithm \\
1: Input $p_{i}(0), R_{i k}, k \in A_{i}, b_{j}(0), r_{i j}, j \in S_{i}$. \\
2: $n \leftarrow 0$, then obtain $\hat{p}_{i}(0)$. \\
3: $p_{i}(n) \leftarrow j \in S_{i}$, then determine $\hat{p}_{i}^{(j)}(n+1), R_{i j}$. \\
4: Subject to: $0<j<N_{i}$, then have $\hat{p}_{i}(n+1)$. \\
5: Broadcast: $\hat{p}_{i}(n+1)$. \\
6: $n \leftarrow n+1$. \\
7: Until $n \leq 100$.
\end{tabular}

\section{Localization Performance evaluation}

For the localization of all nodes of the considered network, a persuasive performance measured indicators is the root of mean squared error (RMSE). Therefore, the RMSE of the estimated position $\hat{p}_{i}$ for a target node is defined as

$$
R M S E=\sqrt{\frac{1}{M} \sum_{i=1}^{M} E\left(\left\|\hat{p}_{i}-p_{i}\right\|^{2}\right)}
$$

A Cramer-Rao lower bound (CRLB)that can be derived according to the probability density function (PDF) of the available pair wise distance measurements given the positions of all nodes [15]. We have the average CRLB given by 


$$
C R L B_{a}=\frac{1}{N} \operatorname{trace}\left[F^{-1}\right]
$$

Where trace is the trace operator, and the Fisher Information Matrix (FIM) $F$ is

$$
F=-E\left[\begin{array}{ccc}
\frac{\partial^{2} \ln f(s)}{\partial p_{1} \partial p_{1}^{T}} & \cdots & \frac{\partial^{2} \ln f(*)}{\partial p_{N} \partial p_{1}^{T}} \\
\vdots & \ddots & \vdots \\
\frac{\partial^{2} \ln f(s)}{\partial p_{1} \partial p_{N}^{T}} & \cdots & \frac{\partial^{2} \ln f(s)}{\partial p_{N} \partial p_{N}^{T}}
\end{array}\right]
$$

Where

$$
\begin{gathered}
f(*)=f\left\{R_{i j}, i, j \in\left[i<j, \gamma_{i j}=1\right] \mid p_{j}\right\} \\
=\frac{1}{\sqrt{\left(2 \pi \sigma_{n}^{2}\right)^{\sum_{i<j i j j=1}^{N} \gamma_{i j}}}} \exp \left(-\sum_{i<j, i, j=1}^{N} \frac{1}{2 \sigma_{n}^{2}} \gamma_{i j}\left(\left\|p_{i}-p_{j}\right\|-R_{i j}\right)^{2}\right)
\end{gathered}
$$

Where $\gamma_{i j}=\gamma_{j i}$ is 1 if node $\mathbf{s}_{i}$ and node $\mathbf{s}_{j}$ are neighbors, and0 otherwise.

$$
-E\left[\frac{\partial^{2} \ln f(*)}{\partial p_{i} \partial p_{i}^{T}}\right]=\frac{1}{\sigma_{n}^{2}} \sum_{i \neq j, j=1}^{N} \gamma_{i j} \frac{\left(p_{i}-p_{j}\right)\left(p_{i}-p_{j}\right)^{T}}{\left\|p_{i}-p_{j}\right\|^{2}}
$$

When $=j=1, \ldots, N$, we have

$$
-E\left[\frac{\partial^{2} \ln f(s)}{\partial p_{i} \partial p_{j}^{T}}\right]=-\frac{1}{\sigma_{n}^{2}} \gamma_{i j} \frac{\left(p_{i}-p_{j}\right)\left(p_{i}-p_{j}\right)^{T}}{\left\|p_{i}-p_{j}\right\|^{2}}
$$

\section{Numerical Simulation Study}

This simulation setting is involved in 10 nodes whose true positions are given in Table 1 , where there are front five nodes to be localized and other five anchors in positioning region in the network. Table 1 True node positions in meters

\begin{tabular}{ccccccccccc}
\hline Coordinates & 1 & 2 & 3 & 4 & 5 & 6 & 7 & 8 & 9 & 10 \\
\hline $\mathrm{x}$ & -40 & 40 & -40 & 40 & 0 & 0 & -20 & 20 & 40 & 0 \\
$\mathrm{y}$ & 40 & 40 & -40 & -40 & 0 & 20 & 0 & 0 & 0 & -20 \\
\hline
\end{tabular}

Fig. 1 shows three algorithms converging to a minimum localization error at different rates after 100 iterations. We consider estimated performance comparison of the proposed algorithm randomly initialized, the proposed algorithm initialized from the correct positions in order to comparison of estimation performance, along with the square root of the CRLB which marked as the algorithm A, algorithm B and algorithm $C$ respectively. The RMSE curve for the algorithm A has a smooth decay with convergence around20 iterations, but its estimation performance has a gap compared with the CRLB. The algorithm B present a fast decay towards a minimum point within10 iterations, and can reach the CRLB in estimation performance. The algorithm $A$ is the last one to reach the lowest RMSE value, and has fast convergence speed when there are around20 average iterations passed at node.

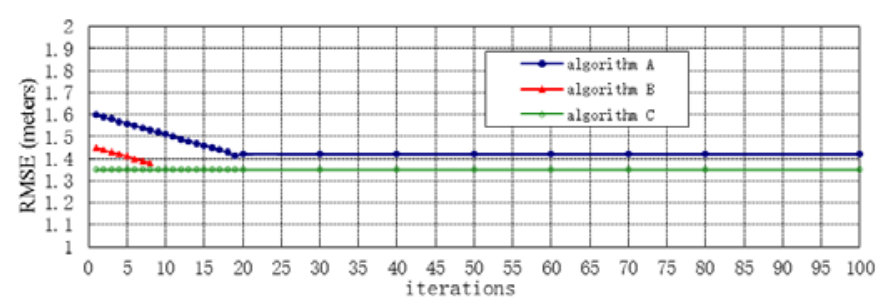

Fig.1. Comparison of RMSE for TDOA measurements as a function of the number of iterations for the algorithm A, algorithm B and algorithm $C$ which used the same set of initial position estimations under random initialization.

Fig.2shows the converged RMSE positioning errors of the three algorithms corresponding to the 
TDOA-based measurement error. The ranging error in the graph is expressed by $10 \log _{10}\left(\sigma^{2}\right)$ length units, and the position error is expressed by $10 \log _{10}(R M S E$ )length units. We can see from the figure that all these algorithms converge and can reach the CRLB. Although the algorithm A always converges globally, there has a gap between its convergence performance and that of CRLB.

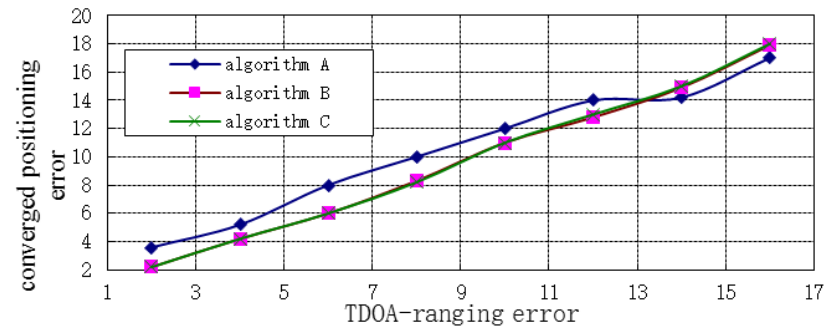

Fig.2. Estimation performance comparison of the converged RMSE positioning errors of the three algorithms corresponding to the TDOA-based measurement error under random initialization.

Fig.3(a) and Fig.3(b) show the simulation results of RMSE positioning errors under the conditions that all algorithms are initialized with the proposed initialization. We can see from the figure that the convergence speed has been significantly improved compared withFig.1. In addition, the converged estimation performance of the algorithm A is slightly better than that acquired by random initialization.

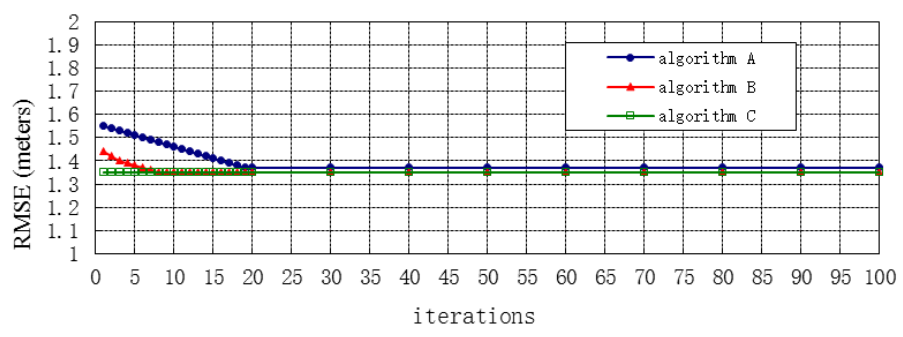

(a)Comparison of RMSE for TDOA measurements as a function of the number of iterations for the three algorithms which used the same set of initial position estimations.

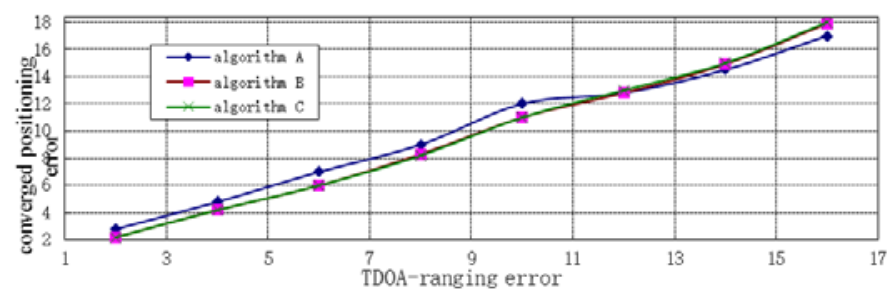

(b)Estimation performance comparison of the converged RMSE positioning errors of the three algorithms corresponding to the TDOA-based measurement error under the proposed initialization.

Fig.3. The simulation results of all algorithms with the proposed initialization.

\section{Conclusion}

In this paper, we proposed a cooperative iterative localization algorithm for localization of target nodes in a network. The algorithm estimates the position of a node using the TDOAs from the anchors and the upgrade nodes whose positions are previously estimated. Analysis and simulation show that when iteratively running at all nodes of the considered network, the proposed estimator is computationally attractive and achieve the best performance. T Future works may include reducing the ranging error, refining cooperative strategy to improve the localization accuracy.

\section{References}

[1] S. Gezici, T. Zhi, G. Giannakis, H. Kobayashi, A. Molisch, H. Poor, and Z. Sahinoglu, "Localization via ultra-wideband radios: A look at positioning aspects for future sensor networks," 
IEEE Signal Process. Mag., vol. 22, pp. 70-84, Jul. 2005.

[2] D. D. McCrady, L. Doyle, H. Forstrom, T. Dempsy, and M. Martorana, "Mobile ranging with low accuracy clocks,” IEEE Trans. Microwave Theory Tech., vol. 48, pp. 951-957, June 2000.

[3] T. Li, A. Ekpenyong, and Y.-F. Huang, "Source localization and tracking using distributed asynchronous sensors,” IEEE Trans. Signal Process., vol. 54, no. 10, pp. 3991-4003, Oct. 2006.

[4] Y. Shen, H. Wymeersch, and M. Z. Win, "Fundamental limits of wide band localization -Part II: A cooperative networks,” IEEE Trans. Inf. Theory, vol. 56, no. 10, pp. 4981-5000, Oct. 2010.

[5] Y. Shen and M. Z. Win, "Fundamental limits of wide band localization-Part I: A general framework,” IEEE Trans. Inf. Theory, vol. 56, no. 10, pp. 4956-4980, Oct. 2010.

[6] N. Decarli, F. Guidi, and D. Dardari, “A novel joint RFID and radar sensor network for passive localization: Design and performance bounds,” IEEEJ. Sel. Topics Signal Process., vol. 8, no. 1, pp. 80-95, Feb. 2014.

[7] T. V. Nguyen, Y. Jeong, H. Shin, and M. Z. Win, "Machine learning for wide band localization,” IEEE J. Sel. Areas Commun., vol. 33, pp. 1-1, July. 2015.

[8] D. Carevic, "Automatic estimation of multiple target positions and velocities using passive TDOA measurements of transients,” IEEE Trans. Signal Process., vol. 55, no. 2, pp. 424-436, Feb. 2007.

[9] K. C. Ho and W. Xu, "An accurate algebraic solution for moving source location using TDOA and FDOA measurements,” IEEE Trans. Signal Process., vol. 52, no. 9, pp. 2453-2463, Sep. 2004.

[10] J. Zheng and Y. C. Wu, "Localization and time synchronization in wireless sensor networks: A unified approach,” in Proc. IEEE Asia Pacific Conf. Circuits Syst., Macao, Nov.-Dec. 2008, pp. 594-597.

[11] M. Vemula, M.F. Bugallo, P.M. Djuric, "Sensor self-localization with beacon position uncertainty, ” Signal Processing., vol. 89, no. 6, pp. 1144-1154, Jun. 2009.

[12] F.K.W. Chan, H.C. So, "Accurate distributed range-based positioning algorithm for wireless sensor networks,” IEEE Transactions on Signal Processing ., vol. 57, no. 10, pp. 4100-4105, May. 2009.

[13] T. Jia and R. M. Buehrer, "On the optimal performance of collaborative position location,” IEEE Trans. Wireless Commun., vol. 9, no. 1, pp. 374-383, Jan. 2010.

[14] J. Cota-Ruiz, J. G. Rosiles, E. Sifuentes, and P. Rivas-Perea, “A low complexity geometric bilateration method for localization in wireless sensor networks and its comparison with least-squares methods,” Sensors, vol. 12, no. 1, pp. 839-862, Jan. 2012.

[15] Y. Shen and M. Z. Win, "On the accuracy of localization systems using wide band antenna arrays, ” IEEE Trans. Commun., vol. 58, no. 1, pp. 270-280, Jan.2010. 